\title{
Comparative Foraging Efficiency of Two Sympatric Jackals, Silver-Backed Jackals (Canis mesomelas) and Golden Jackals (Canis aureus), in the Ngorongoro Crater, Tanzania
}

\author{
S. E. Temu, ${ }^{1}$ C. L. Nahonyo, ${ }^{1}$ and P. D. Moehlman ${ }^{2}$ \\ ${ }^{1}$ Department of Zoology and Wildlife Conservation, College of Natural and Applied Sciences, \\ University of Dar es Salaam, Dar es Salaam, Tanzania \\ ${ }^{2}$ CERC, Columbia University and EcoHealth Alliance, New York City, NY, USA
}

Correspondence should be addressed to S. E. Temu; temusteven25@yahoo.com

Received 22 April 2016; Revised 30 September 2016; Accepted 23 October 2016

Academic Editor: Daniel I. Rubenstein

Copyright (C) 2016 S. E. Temu et al. This is an open access article distributed under the Creative Commons Attribution License, which permits unrestricted use, distribution, and reproduction in any medium, provided the original work is properly cited.

\begin{abstract}
The foraging efficiency of two sympatric species of jackals, silver-backed jackals (Canis mesomelas) and golden jackals (Canis aureus), was studied in the Ngorongoro crater from July 2014 through May 2015. The focal animal observation method was used and individuals of both species were followed as they foraged from morning to evening. Observations of individuals of both jackal species were made from a vehicle using binoculars and a spotting scope. Three major parameters were used for determination of foraging efficiency: distance travelled while foraging, time spent foraging, and amount of food secured in foraging period. The Mann-Whitney $U$ test showed no significant difference $(P>0.05)$ in distance travelled per unit time of foraging between the two species in the dry and wet seasons, respectively. Golden jackals secured a significantly higher amount of food than the silverbacked jackals in the wet season (Mann-Whitney $U$ test, $P<0.05, U=1035.4$ ). Hunting of prey larger than Thomson's gazelle (Eudorcas thomsonii) fawns was not common. Both species mainly fed on smaller prey such as invertebrates and rodents and scavenged opportunistically. Efficient foraging is crucial for both jackal species especially during their breeding season when they are provisioning dependent pups.
\end{abstract}

\section{Introduction}

Searching for enough food to yield energy for survival and reproduction is a major task faced by animals [1]. The foraging process involves decisions (what to eat, where, how long to search, and how to search so as to maximize energy intake per unit effort) in terms of costs and benefits. Costs can be measured in terms of time and energy expended in the act of foraging and benefits in terms of fitness, following Optimal Foraging Theory [2]. The theory, first formulated in 1966 by MacArthur and Pianka [3], states that natural selection favours animals whose behavioural strategies maximize their net energy intake per unit time spent foraging. Such time includes both searching for and handling of prey (i.e., killing and eating).

Mammals forage in a way to achieve a favourable balance between the energy and time expended in securing and metabolizing food and the energy gained from the ingested food. Foraging strategies among animals can help maximize the net rate of energy gain which can result in an increase in fitness. Energy gained by foraging needs to exceed the energy expended. The Optimal Foraging Theory states this searching behaviour is based on a goal to maximize fitness [1].

Energetics in foraging refers to energy expended in food acquisition in terms of costs and benefits. It is expressed as distance per unit time of foraging (costs) and weight/number of preys consumed by animals for their survival (benefits) [4]. A review of the allometry of Canidae and cooperative breeding [5] modelled that the costs and benefits of foraging would affect the parental input to pups. The cost of foraging (e.g., kilometres travelled) and the benefits (e.g., grams of food ingested) may determine the amount of regurgitations given to pups and their subsequent survival (benefit). Pup survival in jackal species also depends on presence of helpers 
(grown offspring) who help by regurgitating food to pups, guarding them when the parents are absent, grooming the pups, and playing with them [6].

Studies on energetics and foraging behaviour have been conducted on other carnivores; Andrews et al. [1] conducted a study on foraging behaviour and energetics of sea lions and found that foraging effort (expressed as distance covered per time spent foraging) by sea lions correlates with pup growth and therefore is indicative of pup survival and female reproductive success. Similarly, a study by Van der Meer et al. [7] indicated that reproductive success of African wild dogs depended on their ability to catch prey while minimizing foraging costs (foraging distance and chase distance) by hunting cooperatively.

Jackals are omnivorous opportunists, feeding on variety of foods including invertebrates, reptiles, birds, small to medium sized mammals, plant materials, and carrion [8]. As relatively small carnivores, jackals could subsist on an invertebrate diet because of their lower absolute energy requirements [9].

Three species of jackals, that is, silver-backed jackal (Canis mesomelas, Schreber 1775), golden jackal (Canis aureus, Linnaeus 1758), and side-striped jackal (Canis adustus, Sundevall 1847), live in the Ngorongoro crater $\left(260 \mathrm{~km}^{2}\right)$ which is part of Ngorongoro Conservation Area (Tanzania). There is limited information on the diet and foraging efficiency of these three jackal species in Ngorongoro crater [10]. This study focused on the foraging efficiencies of two sympatric and diurnally active species of jackals, the silverbacked jackal and the golden jackal, and recorded distance travelled, time spent foraging, and amount of food secured.

\section{Materials and Methods}

2.1. Study Site. The study was carried out in Ngorongoro crater within the Ngorongoro Conservation Area (Figure 1), Tanzania. Ngorongoro crater $\left(3^{\circ} 5^{\prime}-3^{\circ} 15^{\prime} \mathrm{S} ; 35^{\circ} 25^{\prime}-35^{\circ} 40^{\prime} \mathrm{E}\right)$, which covers an area of $260 \mathrm{~km}^{2}$, has one of the highest densities of predators in the world including lions (Panthera leo), spotted hyenas (Crocuta crocuta), cheetah (Acinonyx jubatus), leopards (Panthera pardus), and jackals (Canis sp.) [10]. There are two seasons: the dry season (June through September) and the rainy season (October through May). The crater receives an annual rainfall between $600 \mathrm{~mm}$ and $900 \mathrm{~mm}$ and temperature is between $24^{\circ} \mathrm{C}$ and $30^{\circ} \mathrm{C}$ [11].

2.2. Data Collection. Data were collected from July 26, 2014, to May 18, 2015, covering both dry and wet seasons. The focal animal observation method was used [12]. Individuals of both species were followed as they foraged from morning to evening. Observations were made from a vehicle using binoculars and a spotting scope. Follows were per species, per individual, or per a pair per day. All activities performed by an individual/pair under observation were recorded with time, GPS coordinates, habitat, and odometer readings.

(i) Distance Travelled per Unit Time of Foraging (Foraging Cost). The Land Rover odometer (tenths of kilometres) was used in estimating the distance travelled by individuals. Per focal animal follow the total numbers of hours, kilometres, and food items and their estimated weight were recorded. In addition to foraging data other activities including social behaviour, marking, and defecating were recorded. Data on foraging was recorded from the first sighting of the individual until the end of the follow. Time spent resting was not included in the foraging analyses.

(ii) Foraging Efficiency (Foraging Benefit). Number and weight of prey items eaten by the focal individual, for example, insects, rodents, and carrion, were recorded. Estimation of weights of prey items was based on known weights from literature. Dung beetles were the main insects consumed (approximately 5 grams) [13]. Rodents weighed between 70 and 130 grams [14]. Estimates of carrion consumed were done consistently according to size of meat that was consumed, for example, $0.5 \mathrm{~kg}, 2 \mathrm{~kg}$, and $3 \mathrm{~kg}$.

Foraging efficiency was expressed as weights of food consumed (all food items combined) per kilometre per hour of foraging, that is, foraging benefit-cost ratio, and foraging efficiency was compared within and between dry and wet seasons for the two jackal species.

\subsection{Data Analyses}

(i) Distance Travelled per Unit Time of Foraging (Foraging Cost). Mean distance (kilometres) per unit time (hours) per species per season was computed and presented in Table 1. Data were tested for normality and were found to be not normally distributed. A Mann-Whitney $U$ test was used for testing the difference in kilometres travelled per hour of foraging per season by each species.

(ii) Foraging Efficiency. Mean weights (grams) of all food consumed per kilometre and per hour were computed. The Mann-Whitney $U$ test was used for testing the difference in weight of food secured per kilometre and per time of foraging, that is, foraging efficiency between species and between seasons after testing the data for normality (data were not normally distributed).

\section{Results}

3.1. Distance Travelled per Unit Time of Foraging and Foraging Efficiency of the Two Jackal Species. A total of 131 hours and 223.8 hours were spent observing silver-backed jackals in dry season (July-September, 2014) and in wet season (JanuaryMay, 2015), respectively. A total of 125.1 hours and 227.8 hours were spent observing golden jackals in dry season and in wet season, respectively. Silver-backed jackals spent 79.65 hours while golden jackals spent 68.90 hours of this time foraging in the dry season. In the wet season, silver-backed jackals and golden jackals spent 59.63 hours and 97.43 hours for foraging. Silver-backed jackals were found in medium to long grasslands. Golden jackals were in adjacent short grasslands. Results (including $P$ values) are summarized in Table 1.

\section{Discussion}

The time and distance covered while finding and securing prey are the determinants of the energy expended (costs). 


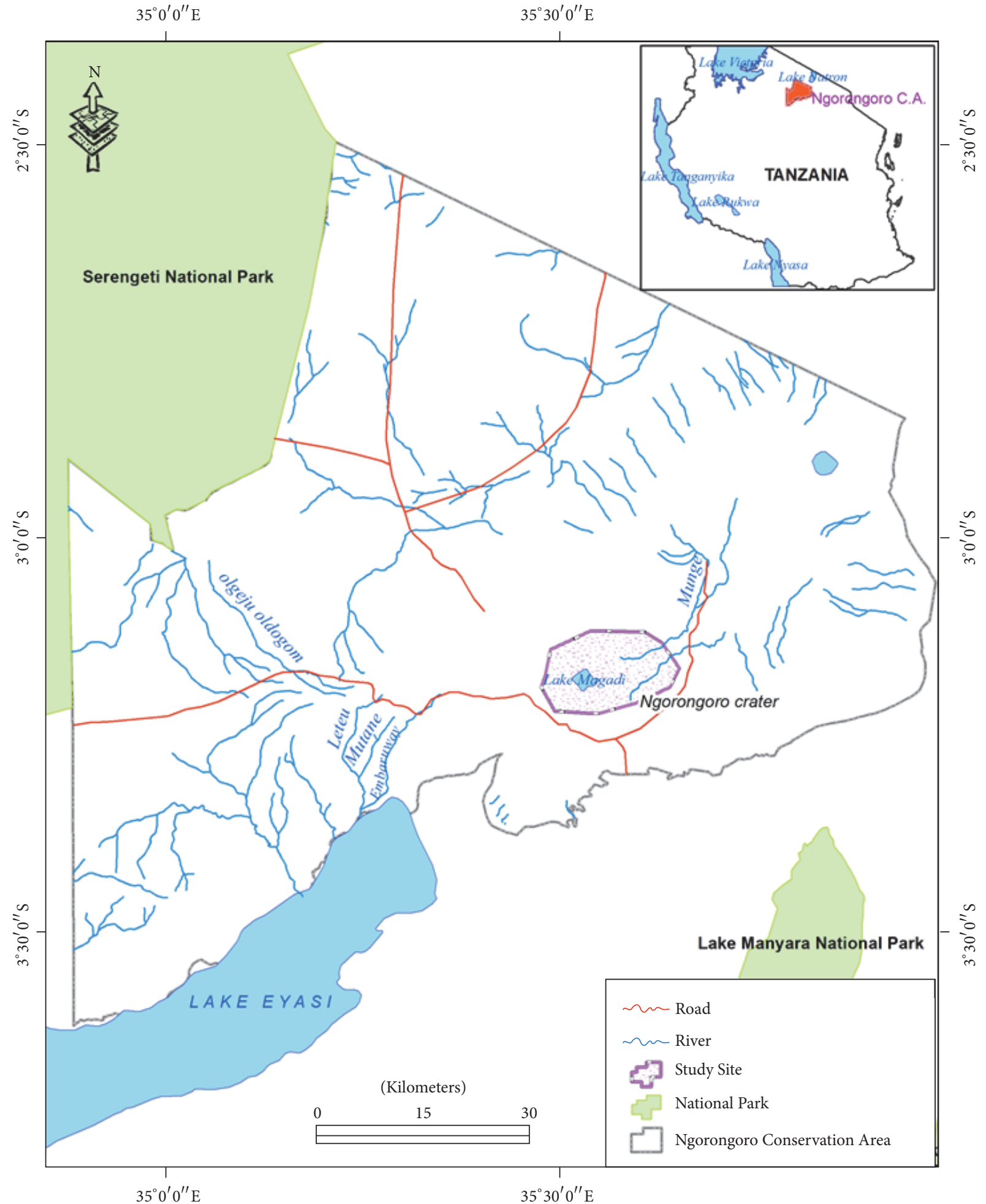

Figure 1: Map of Ngorongoro Conservation Area. Source: Cartographic and Map Curating Unit, Geography Department, University of Dar es Salaam, 2015.

Energy gained (benefits) is measured by the weight of the food ingested. Efficient foraging means less energy expenditure and more food secured per unit time and distance for the focal jackal and is important for survival. When jackals are raising pups their food intake can influence their ability to provide adequate nutrition for their offspring in terms of milk and regurgitated food. Dietary efficiency influences the milk quality in carnivores which also determines growth rate, 
TABLE 1: A summary of the distance travelled per unit time $(\mathrm{km} / \mathrm{hour})$ and the foraging efficiency (weight of food consumed per km and per hour) by the two jackals species in the dry and wet seasons.

\begin{tabular}{|c|c|c|c|c|c|c|c|c|}
\hline & \multicolumn{3}{|c|}{ Silver-backed jackal } & \multicolumn{3}{|c|}{ Golden jackal } & \multicolumn{2}{|c|}{$\begin{array}{l}\text { Silver-backed versus golden } \\
\text { jackal }\end{array}$} \\
\hline & Dry season & Wet season & $P$ value & Dry season & Wet season & $P$ value & $\begin{array}{c}\text { Dry season } \\
(P \text { value })\end{array}$ & $\begin{array}{c}\text { Wet season } \\
(P \text { value })\end{array}$ \\
\hline $\mathrm{km}$ of foraging & 128.45 & 110.85 & & 109.36 & 158.55 & & & \\
\hline Hours of foraging & 79.65 & 59.63 & & 68.90 & 97.43 & & & \\
\hline $\mathrm{km} / \mathrm{hr}$ of foraging & 1.61 & 1.86 & $\begin{array}{c}0.7664 \\
(U=483)\end{array}$ & 1.59 & 1.63 & $\begin{array}{c}0.5068 \\
(U=344)\end{array}$ & $\begin{array}{c}0.2911 \\
(U=206)\end{array}$ & $\begin{array}{c}0.3266 \\
(U=13112)\end{array}$ \\
\hline $\begin{array}{l}\text { Grams of food } \\
\text { consumed per km } \\
(\mathrm{g} / \mathrm{km})\end{array}$ & 18.68 & 59 & $\begin{array}{c}0.2268 \\
(U=7368)\end{array}$ & 52.69 & 96.88 & $\begin{array}{c}0.1335 \\
(U= \\
5432)\end{array}$ & $\begin{array}{c}0.1073 \\
(U=8580)\end{array}$ & $\begin{array}{c}{ }^{*} 0.0019(U= \\
9816)\end{array}$ \\
\hline $\begin{array}{l}\text { Grams of food } \\
\text { consumed per } \\
\text { hour of foraging } \\
(\mathrm{g} / \mathrm{hr})\end{array}$ & 30.12 & 109.67 & $\begin{array}{c}0.1841 \\
(U=7296)\end{array}$ & 83.63 & 157.65 & $\begin{array}{c}0.1026 \\
(U= \\
5368)\end{array}$ & $\begin{array}{c}{ }^{*} 0.0235(U= \\
3256)\end{array}$ & $\begin{array}{c}{ }^{*} 0.0019(U= \\
9816)\end{array}$ \\
\hline
\end{tabular}

Note. All statistical tests were performed using Mann-Whitney $U$ test. $U$-values are given in the brackets. * indicates significant difference.

litter size, and birth weight [15]. A predator's search time and distance travelled are influenced by the distribution of prey and the quantity and quality of diet are important for survival and reproduction [16].

The higher foraging efficiency for golden jackal in the wet season as compared to silver-backed jackal may be linked to the breeding season for this species. The wet season is the typical breeding season for the golden jackals in SerengetiNgorongoro ecosystem whereas the dry season is a breeding season for the silver-backed jackals $[8,17,18]$. All adult golden jackals that were followed in the wet season were raising pups. The golden jackals also had a significantly higher foraging efficiency in the dry season. This may indicate that golden jackals are generally more efficient foragers than silver-backed jackals. Most of the silver-backed jackals that were followed in the dry season had pups but the results indicate that they secured more food in the wet season, although the difference was not significant. During the wet season food may be more available and energetically cheaper to secure. Further research is needed to determine if silverbacked jackal foraging efficiency is better in the wet season. This would also raise the question as to why silver-backed jackals have their pups in the dry season.

The diet of jackal species changes with food availability [19]. During the wet season both species hunted and fed on Thomson's gazelle fawns. Although the fawn's mother defended the fawn, the jackal pairs of both species cooperatively hunted and successfully killed fawns. Additionally, golden jackals were observed to hunt and feed on Abdim's storks (Ciconia abdimii) which were abundant in the short grasslands. Wildebeest (Connochaetes taurinus) tend to calve in the short grassland and golden jackals fed on the abundantly available placentas. Both species were observed to hunt for cape hare but the attempts were not successful.

The two jackal species are of similar size (5-9 kg) [5]. Both species frequently consumed invertebrates, small mammals, and vegetable matter. Feeding on these smaller prey items may be due to their availability and lower energetic costs.
Focal individuals/pairs of both species were observed to opportunistically inspect dung of large herbivores such as elephants (Loxodonta africana), buffalo (Syncerus caffer), zebras (Equus quagga), and wildebeests (Connochaetes taurinus) and easily secured and ate dung beetles.

Both jackal species are small carnivores and the largest observed prey size was an adult female Thomson's gazelle $(15-25 \mathrm{~kg})$. Their major prey species were Thomson's gazelle fawns (birth weight: $2-3 \mathrm{~kg}$ ) and rodents (70-130 g). Larger prey like Thomson's gazelle adults and fawns were hunted cooperatively which can increase foraging success [20, 21].

Scavenging may also be a good source of food, but it involves risk and energetic costs. Large carnivores like lions and spotted hyenas defend the carcass from approaching jackals and other intruders such as vultures and eagles. Competition at carcasses also occurs between the two jackal species and between jackals and vultures. At carcasses, jackals from adjoining territories will trespass and attempt to scavenge. When a large carcass (e.g., buffalo, wildebeest, or zebra) is detected, jackals of both species may have to wait until the larger carnivores are finished with eating. Scavenging can provide large amounts of meat that are energetically less costly to consume. Scavenging provided $38.8 \%$ and $87.2 \%$ of the observed food consumption for silver-backed and golden jackals in the dry season. Scavenging was a less important source of food for golden jackal (11.1\%) in the wet season when predation on Abdim's storks provided the most food (35.1\%). Scavenging remained important for silver-backed jackal $(27.5 \%)$ in the wet season.

Carbone et al. [22] proposed a model that carnivores weighing less than $21.5 \mathrm{~kg}$ would feed on prey that was less than $45 \%$ of their body weight. Normally both golden and silver-backed jackals prey on vertebrates like rodents and hares that are $1.4 \%$ and $29 \%$ of their body weight, respectively. Both species of jackals are omnivores and rely on invertebrates and small vertebrates as their main source of food. 


\section{Conclusion}

Opportunistic foraging, cooperative hunting, scavenging, feeding on invertebrates and other smaller food items, and caching of food are among the strategies used by medium sized canids such as jackals to increase foraging success and minimize foraging costs. Both species are opportunistic foragers and feed on whatever food is available and easily obtained. Both golden jackals and silver-backed jackals secured more food during the wet season than during the dry season. The seasonal differences in amount of food secured by each species reflect availability in their home ranges and relate to their different breeding seasons. Efficient foraging is very important for adults' survival and the survival of their pups.

\section{Competing Interests}

The authors declare that they have no competing interests.

\section{Acknowledgments}

The authors thank Tanzania Wildlife Research Institute (TAWIRI) and the Ngorongoro Conservation Area Authority (NCAA) for providing the research permission that made this work possible. They are indebted to Mr. Pascal Joachim for his assistance in the field and Dr. Bruno Nyundo from the Department of Zoology and Wildlife Conservation for assisting in scat analysis in the lab.

\section{References}

[1] R. D. Andrews, D. G. Calkins, R. W. Davis, B. L. Norcross, K. Peijnenberg, and A. W. Trites, "Foraging Behavior and Energetics of Adult Female Steller Sea Lions," 2001, http://www .zoology.ubc.ca/ consort/pdfs/steller_sea_lions_foraging.pdf.

[2] T. M. Smith and R. L. Smith, Elements of Ecology, Pearson Benjamin Cummings, San Francisco, Calif, USA, 7th edition, 2009.

[3] R. H. MacArthur and E. R. Pianka, "On optimal use of a patchy environment," The American Naturalist, vol. 100, no. 916, pp. 603-609, 1966.

[4] R. D. Andrews, D. G. Calkins, R. W. Davis, B. L. Norcross, K. Peijnenberg, and A. W. Trites, "Foraging behavior and energetics of adult female steller sea lions," in Steller Sea Lion Decline: Is It Food II, pp. 19-22, Alaska Sea Grant College Program, 2001, http://www.zoology.ubc.ca/ consort/pdfs/ steller_sea_lions_foraging.pdf.

[5] P. D. Moehlman and H. Hofer, "Cooperative breeding, reproductive suppression, and body mass in canids," in Cooperative Breeding in Mammals, N. Solomon and J. French, Eds., pp. 76128, Cambridge University Press, Cambridge, Mass, USA, 1996.

[6] P. D. Moehlman, "Jackal helpers and pup survival," Nature, vol. 277, no. 5695, pp. 382-383, 1979.

[7] E. Van der Meer, G. S. A. Rasmussen, J. Muvengwi, and H. Fritz, "Foraging costs, hunting success and its implications for African wild dog (Lycaon pictus) conservation inside and outside a protected area," African Journal of Ecology, vol. 52, no. 1, pp. 6976, 2014.
[8] P. D. Moehlman, "Ecology of cooperation in canidae," in Ecological Aspects of Social Evolution, D. Rubinstein and R. Wrangham, Eds., Princeton University Press, Princeton, NJ, USA, 1986.

[9] C. Carbone, G. M. Mace, S. C. Roberts, and D. W. Macdonald, "Energetic constraints on the diet of terrestrial carnivores," Nature, vol. 402, no. 6759, pp. 286-288, 1999.

[10] L. A. Swanson, Ngorongoro conservation area: spring of life [M.S. thesis], University of Pennsylvania, Philadelphia, Pa, USA, 2007.

[11] O. Marttila, The Great Savanna: The National Parks of Tanzania and other Key Conservation Areas, Auris Publishers, Rauha, Finland, 2011.

[12] J. Altmann, "Observational study of behavior: sampling methods," Behaviour, vol. 46, no. 3-4, pp. 227-267, 1974.

[13] D. S. Kettle, Medical and Veterinary Entomology, Centre for Agriculture and Biosciences International (CABI), Wallingford , UK, 1990.

[14] L. Granjon, A. Bekele, and J.-F. Ducroz, "Genus Arvicanthis: grass rats," in The Mammals of Africa. Volume 3. Rodents, Hares and Rabbits, vol. 3, Happold DCD, Bloomsbury, London, UK, 2013.

[15] M. E. Sunquist and F. C. Sunquist, "Ecological constraints on predation by large felids," in Carnivore Behaviour, Ecology, and Evolution, J. L. Gittleman, Ed., Cornell University Press, 1989.

[16] M. E. Sunquist and F. C. Sunquist, "Ecological constraints on predation by large felids," in Carnivore Behaviour, Ecology, and Evolution, Gittleman JL, Cornell University Press, Ithaca, NY, USA, 1989.

[17] P. D. Moehlman, "Socioecology of silver-backed and golden jackals (Canis mesomelas and Canis aureus)," in Recent Advances in the Study of Mammalian Behaviour, J. F. Eisenberg and D. J. Kleiman, Eds., Special Publication no. 7, pp. 423-453, The American Society of Mammalogists, 1983.

[18] P. D. Moehlman, "Social organization in jackals," in Exploring Animal Behaviour, P. W. Sherman and J. Alcock, Eds., pp. 209218, Sinauer Associates, Sunderland, Mass, USA, 1993.

[19] R. Walton and O. Damien, Mammalian Species. Canis Mesomelas, American Society of Mammalogists no. 715, 2003.

[20] M. W. Fox, The Wild Canids. Their Systematics, Behavioural Ecology and Evolution, Van Nostrand Reinhold Ltd, New York, NY, USA, 1975.

[21] J. Lamprecht, "On diet, foraging behaviour and interspecific food competition of jackals in the Serengeti National Park, East Africa," Zeitschrift für Säugetierkunde, vol. 43, pp. 210-223, 1978.

[22] C. Carbone, G. M. Mace, S. C. Roberts, and D. W. Macdonald, "Energetic constraints on the diet of terrestrial carnivores," Nature, vol. 402, no. 6759, pp. 286-288, 1999. 

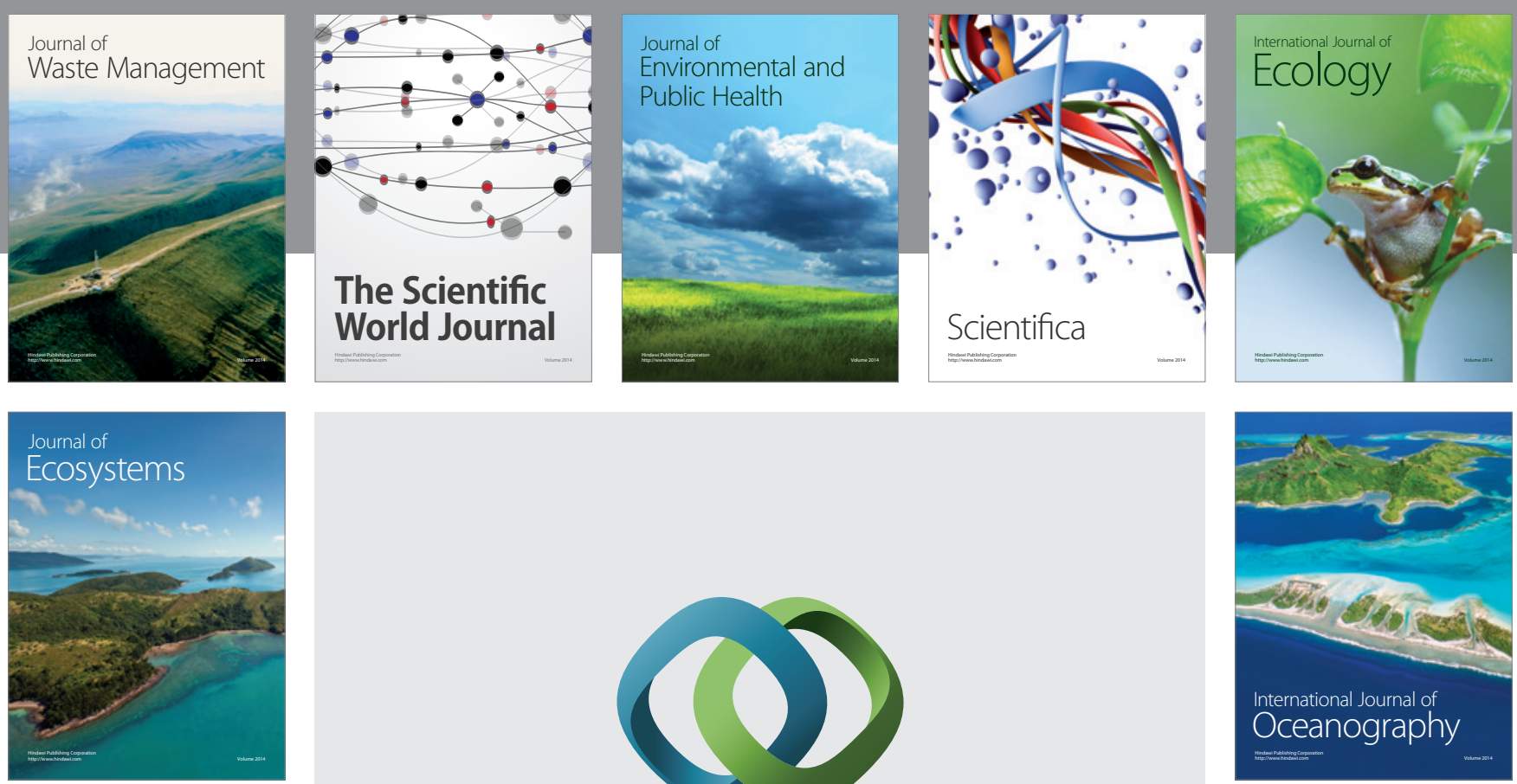

The Scientific World Journal
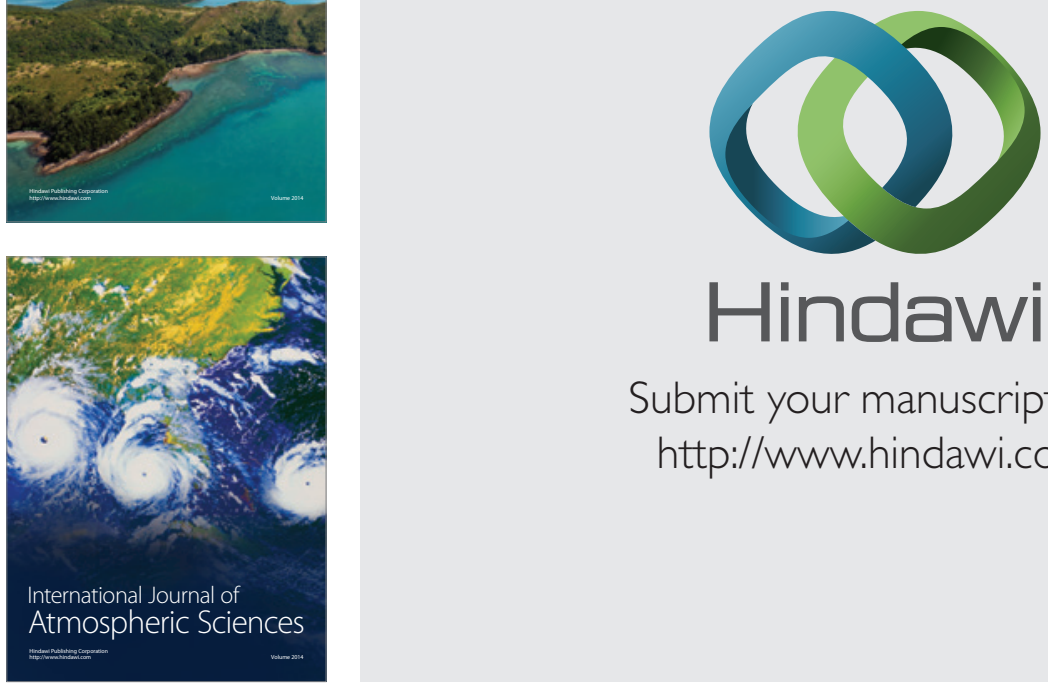

\section{Hindawi}

Submit your manuscripts at

http://www.hindawi.com
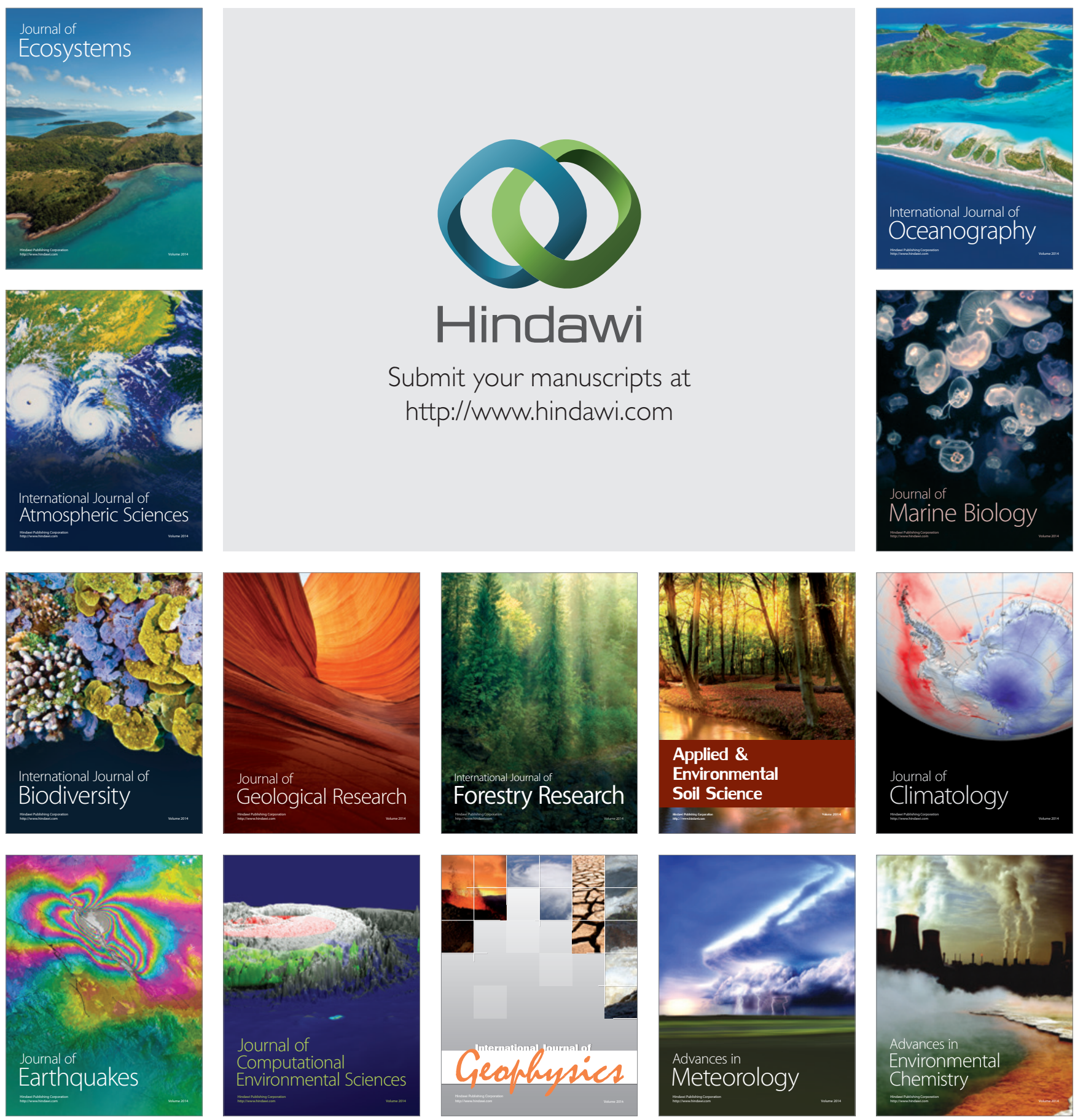